\title{
Théologiques
}

\section{Le début de l'univers vu par un physicien}

\section{Gilles Beaudet}

Volume 2, numéro 1, mars 1994

Création et créativité

URI : https://id.erudit.org/iderudit/602400ar

DOI : https://doi.org/10.7202/602400ar

Aller au sommaire du numéro

Éditeur(s)

Faculté de théologie de l'Université de Montréal

ISSN

1188-7109 (imprimé)

1492-1413 (numérique)

Découvrir la revue

Citer cette note

Beaudet, G. (1994). Le début de l'univers vu par un physicien. Théologiques, 2(1), 103-107. https://doi.org/10.7202/602400ar d'utilisation que vous pouvez consulter en ligne.

https://apropos.erudit.org/fr/usagers/politique-dutilisation/ 


\section{Le début de l'univers vu pas un physicien}

\section{Gilles BEAUDET \\ Département de physique \\ Université de Montréal}

Toutes les sociétés humaines, même les plus primitives, se sont donné une vision du monde, de leur monde. Il est tout à fait naturel pour l'homme de s'interroger sur ses origines et sur son avenir : nous voulons savoir d'où nous venons et où nous allons. Il n'est donc pas étonnant que notre société, elle aussi, avec sa forte composante technologique et scientifique ait essayé de répondre à ces mêmes questions, en faisant appel au bagage impressionnant de connaissances accumulées au cours des siècles. Les origines que nous allons considérer dans les pages suivantes sont celles de l'univers tout entier, celles que les scientifiques se gardent bien d'appeler création puisque, comme nous le verrons, ils n'atteignent pas et n'atteindront probablement jamais la connaissance du temps zéro.

La théorie qui prévaut aujourd'hui dans le monde scientifique est la théorie de l'explosion initiale, mieux connue sous le nom de la théorie du Big Bang. Elle est de facture assez récente, ayant pris forme au début du siècle, mais n'ayant obtenu véritablement ses lettres de créances que vers le milieu des années soixante. C'est une théorie toute simple qui étonne toujours par son succès. Ce n'est pas qu'elle manque d'opposition; elle rencontre parfois des difficultés sérieuses. Il demeure cependant que personne n'a encore pu lui substituer une autre théorie qui rendrait compte des faits d'une manière aussi élégante.

Il y a trois raisons empiriques qui supportent l'idée du Big Bang: l'expansion de l'univers, la radiation du fond du ciel et la présence de l'hydrogène lourd dans l'univers. Avant d'expliquer pourquoi ces trois faits expérimentaux sont importants, il est bon de se rappeler comment l'idée d'une explosion initiale a pu apparaître.

Il faut retourner à Isaac Newton au XVII e siècle pour observer la première percée dans notre compréhension de l'univers. Newton, comme 
bien d'autres de son temps, essayait de comprendre et d'expliquer le mouvement des planètes. En observant une pomme tombant de son arbre, il eut une idée tout à fait originale : les lois de la physique terrestre sont les mêmes que celles qui s'appliquent au mouvement des planètes. Auparavant, les gens croyaient que les lois du ciel (i.e. tout ce qui se trouvait hors de la surface de la terre) étaient tout à fait indépendantes du monde imparfait dans lequel nous vivons. Newton affirma au contraire que la force qui fait tomber la pomme est la même que celle qui fait tourner la terre autour du soleil. Cela n'est pas évident à première vue puisque la trajectoire de la pomme est rectiligne et celle de la terre, courbe. C'est pourtant bel et bien la gravité qui s'exerce dans les deux cas, les différences étant expliquées par les vitesses initiales.

Après Newton, les sciences dites naturelles (physique, chimie, biologie, etc.) font des progrès énormes comparativement aux siècles précédents. Pour le sujet qui nous intéresse cependant, la fin du XIX ${ }^{\mathrm{e}}$ siècle verra apparaître une impasse : la mesure de la vitesse de la lumière. Celle-ci semblait indépendante du système de références. Albert Einstein proposa alors l'existence d'une vitesse limite supérieure dans l'univers, une vitesse égale à celle de la lumière et l'équivalence des masses gravitationnelles et d'inertie. Il y eut plusieurs conséquences à ces hypothèses. Premièrement, le temps et l'espace devenaient liés l'un à l'autre et la notion de simultanéité prenait un sens bien différent. Deuxièmement, la masse, l'énergie et la vitesse de la lumière s'unissent dans l'équation $\mathrm{E}=\mathrm{mc}^{2}$. Troisièmement, et ceci est peut-être moins connu, les équations d'Einstein conduisent à un univers non statique. Einstein lui-même ne retint pas cette idée puisqu'il n'avait aucun fait expérimental pouvant la supporter.

Durant ce temps, les astronomes amélioraient sensiblement leurs instruments et l'image d'un univers immensément grand par rapport à l'échelle humaine se construisait laborieusement. Grâce à une technique développée en laboratoire, ils avaient trouvé que les éléments chimiques de base dans les étoiles étaient les mêmes que sur la terre et que le monde matériel, qu'il fût poussière d'étoiles ou poussière terrestre, démontrait une unité totale. Vers 1910, ils avaient repéré les galaxies, ces îlots-univers composés de milliards de soleils. Nous-mêmes, habitons une galaxie que nous appelons la voie lactée, à cause de son apparence dans le ciel étoilé. À peu près tout ce qui se voit à l'oeil nu se trouve dans cette voie lactée.

Et voilà que Hubble, astronome américain travaillant sur un nouveau télescope en Californie, découvre que toutes les galaxies s'éloignent de la 
nôtre avec des vitesses d'autant plus grandes qu'elles sont plus éloignées. La relation entre la vitesse et la distance est linéaire dans le sens qu'une distance deux fois plus grande indique une vitesse deux fois plus grande. Il était facile alors, en divisant la distance par la vitesse, d'obtenir un «âge» pour l'univers, c'est-à-dire le temps écoulé depuis la séparation de ces galaxies. Cet âge mesuré de l'univers était de l'ordre de dizaines de milliards d'années et dépendait de la précision des mesures de distance.

L'idée de l'explosion initiale ne fut cependant pas acceptée d'emblée par cette découverte. La mesure des vitesses et des distances en astronomie ne se fait pas directement, mais indirectement (les mesures directes étant impossibles à cause des distances énormes impliquées). Les vitesses se mesurent, entre autres, par le décalage vers le rouge de la lumière (l'objet paraît plus rouge qu'il ne l'est en réalité). D'autres phénomènes physiques peuvent produire un rougissement de la lumière et les opposants au Big Bang pouvaient argumenter, avec raison d'ailleurs, que la preuve n'était pas suffisante.

Il fallait autre chose pour soutenir la théorie : une preuve convaincante est venue quelque quarante ans plus tard par la découverte de la radiation du fond du ciel. Le raisonnement est le suivant : s'il y a eu explosion initiale et expansion de l'univers, la température très élevée du début a dû décrôitre depuis tout ce temps pour se situer aujourd'hui autour de -270 degrés Celsius. C'est l'effet réfrigérateur : un gaz en expansion se refroidit. Aussi surprenant que cela puisse paraître, cet effet est mesurable. Nous recevons une radiation dans les ondes micro-métriques de toutes les directions autour de la terre. Cette radiation nous indique la température du Big Bang actuel. C'est comme si nous étions au centre d'une immense sphère dont les parois émettraient une radiation. Cette radiation est tout à fait uniforme : les variations observées tout récemment ne dépassent pas une partie dans cent mille.

Cette découverte, prédite par calcul quelques années auparavant, a été effectuée par hasard par deux savants américains, Penzias et Wilson, qui cherchaient autre chose. À partir de ce moment, la théorie du Big Bang est devenue la théorie admise par la communauté. Une dizaine d'années plus tard, les chercheurs qui tentaient d'expliquer l'abondance relative des éléments dans l'univers, rencontraient des difficultés avec l'hydrogène lourd (l'élément qui remplace l'hydrogène ordinaire dans la composition de l'eau lourde). Cet élément est difficile à fabriquer et facile à détruire. Le soleil n'en possède pratiquement pas, alors que sur la terre, on le retrouve dans l'eau ordinaire dans une proportion d'une partie par dix mille, ce qui est très élevé. Pour le produire en aussi grande quantité, il 
faut un milieu explosif : aussitôt après sa production, il est expulsé; ce qui l'empêche d'être détruit dans les hautes températures ambiantes. Actuellement, la théorie du Big Bang peut expliquer l'abondance de l'hydrogène lourd dans l'univers avec une simplicité remarquable. Dans l'esprit de plusieurs scientifiques, cet autre succès confirma la validité de la théorie.

Où en sommes-nous aujourd'hui? Ce qu'il faut comprendre, c'est qu'en allant vers le passé, nous nous dirigeons vers des régions où la température est de plus en plus élevée. Aujourd'hui, la température est de -270 degrés Celsius. En analysant la radiation du fond du ciel, nous reculons jusqu'à une température de 3000 degrés Celsius environ 100000 ans après le début. L'hydrogène lourd s'est synthétisé à une température de un milliard de degrés et ce, à une seconde du début. Les physiciens des particules sont intéressés par des températures de mille milliards de degrés et ce sont eux qui, aujourd'hui, font le plus de recherche sur la théorie du Big Bang. C'est la rencontre de l'extrêmement grand - la cosmologie et de l'extrêmement petit - la physique des particules. Comme vous l'avez sans doute déjà noté, la température et le temps sont à l'inverse l'un de l'autre et nous ne pourrons pas plus atteindre une température infinie qu'un temps zéro. Nous ne pouvons reculer au-delà de ce que nos connaissances de la physique nous permettent. Selon les chercheurs en physique des particules cependant, c'est dans les tous premiers instants de l'univers que les lois de la physique et le rapport entre les différentes forces de la nature se sont établis, et c'est dans cette direction que les recherches doivent s'orienter pour la compréhension de la physique fondamentale.

Je voudrais maintenant répondre à certaines questions soulevées chaque fois par cetre théorie.

a) Où se trouve le centre de l'univers? En fait, les gens demandent où se trouve le centre de l'explosion. La réponse, c'est que le centre est en même temps partout et nulle part. Nous ne devons pas concevoir l'explosion centrale comme quelque chose qui se produit au loin. Nous sommes exactement au centre de cette explosion, puisque ce sont tous les points de l'espace qui explosent. Cependant, quelqu'un placé ailleurs voit exactement les choses de la même façon que nous et peut en déduire lui aussi qu'il est au centre. Le centre de l'univers est donc partout et nulle part.

b) Qu'est-ce qu'il y avait avant? Les physiciens ne peuvent répondre à cette question. Tout ce que nous pouvons faire, c'est reculer dans le temps de plus en plus, jusqu'à la limite de nos connaissances. Nous ne pouvons rien dire d'autre: la question n'a pas de sens physique. 
c) Est-ce que l'univers est infini? Notre «univers» à nous est fini: il est limité par la distance traversée par la lumière depuis le début de l'univers, soit environ 15 milliards d'années. Nous appelons "horizon", cette distance au-delà de laquelle nous ne pouvons plus recevoir d'informations. Il est toujours possible cependant que l'univers soit infini, mais nous ne pourrons pas le savoir puisque l'information venant de l'autre côté de notre horizon n'a pas eu le temps de nous parvenir.

d) L'univers continuera-t-il de s'étendre? Cela dépend de la quantité de matière contenue dans l'univers. S'il y en a suffisamment, la gravité sera telle que l'expansion peut être arrêtée et même renversée. La matière visible n'est pas suffisante cependant : il en faudrait dix fois plus. De là vient l'idée de la masse manquante de l'univers puisque certaines hypothèses proposées par les spécialistes des particules élémentaires exigent une densité de matière juste suffisante pour arrêter l'expansion sans pouvoir cependant initier une contraction. Ceci reste à démontrer et nous devrions en savoir davantage dans quelques années.

e) Y a-t-il des problèmes que la théorie actuelle du Big Bang ne peut expliquer? La réponse est oui. Il aurait été surprenant qu'une théorie aussi simple ne rencontre pas de difficultés. L'une de celles-là est la grande uniformité de la radiation du fond du ciel. Comment deux parties opposées du ciel peuvent-elles montrer la même température puisque l'information n'a pas eu le temps de voyager entre un lieu et l'autre? L'explication proposée : rien ne garantit que l'univers s'est toujours étendu au même rythme. Il a très bien pu exister, au début, des périodes d'expansion plus rapides ou plus lentes. En fait, il y a moyen de répondre à des difficultés en compliquant le modèle. Peut-être qu'un jour nous n'y parviendrons plus, nous verrons alors ce qu'il faudra changer.

Et la création? La physique qui s'intéresse au monde matériel ne peut donner comme réponses que celles basées sur la matière. Cependant, il y a amplement place à réflexion. La vie sur terre dépend d'une manière très sensible sur les rapports entre les forces fondamentales de la nature et ces rapports ont été établis dès le premier millionième de seconde de l'univers. Or, le moindre changement dans ces rapports peut conduire à un univers totalement différent où la vie, telle que nous la connaissons, n'aurait pu être possible. L'évolution inhérente à la vie a finalement donné l'homme et sa capacité d'abstraction. Certains se sont demandé si notre univers n'était pas une machine à produire la conscience... Sommes-nous le fruit du hasard?... 